2 Impact of Salivary and Pancreatic Amylase Gene Copy

3 Numbers on Diabetes, Obesity, and Functional Profiles of

4 Microbiome in Northern Japanese Population

\title{
6 Authors and Affiliations
}

7 Takanori Hasegawa $^{1^{*}}$, Masanori Kakuta ${ }^{2}$, Rui Yamaguchi ${ }^{2}$, Noriaki Sato ${ }^{3}$, Tatsuya

8 Mikami $^{4}$, Koichi Murashita ${ }^{5}$, Shigeyuki Nakaji ${ }^{6}$, Ken $\operatorname{Itoh}^{7}$, Seiya Imoto ${ }^{1 *}$.

${ }^{1}$ Health Intelligence Center, The Institute of Medical Science, The University of Tokyo,

11 4-6-1 Shirokanedai, Minato-ku, Tokyo, 108-8639, Japan

$12{ }^{2}$ Human Genome Center, The Institute of Medical Science, The University of Tokyo,

13 4-6-1 Shirokanedai, Minato-ku, Tokyo, 108-8639, Japan

$14{ }^{3}$ Department of Biomedical Data Intelligence, Graduate School of Medicine, Kyoto

15 University, 54 Shogoin Kawahara-cho, Sakyo-ku, Kyoto, 606-8507, Japan

16 Innovation Center for Health Promotion, Hirosaki University Graduate School of

17 Medicine, 5 Zaifu-cho, Hirosaki, Aomori, 036-8562, Japan

$18{ }^{5} \mathrm{COI}$ Research Initiatives Organization, Hirosaki University, 5 Zaifu-cho, Hirosaki,

19 Aomori, Japan

$20{ }^{6}$ Department of Social Medicine, Hirosaki University Graduate School of Medicine, 5

21 Zaifu-cho, Hirosaki, Aomori, 036-8562, Japan

$22 \quad{ }^{7}$ Department of Stress Response Science, Hirosaki University Graduate School of

23 Medicine, 5 Zaifu-cho, Hirosaki, Aomori, 036-8562, Japan

\section{*Corresponding author}

26 E-mail addresses: t-hasegw@ims.u-tokyo.ac.jp (T. Hasegawa), mkakuta@ims.u-tokyo.ac.jp (M. Kakuta),

27 ruiy@ims.u-tokyo.ac.jp (R. Yamaguchi),nori5030@kuhp.kyoto-u.ac.jp (N. Sato),

28 tmika@hirosaki-u.ac.jp (T. Mikami), murasita@hirosaki-u.ac.jp (K. Murashita), nakaji@hirosaki-u.ac.jp

29 (S. Nakaji), itohk@hirosaki-u.ac.jp (K. Itoh), imoto@ims.u-tokyo.ac.jp (S. Imoto).

Abstract

32 Amylase genes reside in a structurally complex locus, and their copy numbers vary

33 greatly, especially among agricultural races. Amylase genes seem to shape the

34 metabolic response to dietary starch, and several studies have reported their association

35 with obesity. Besides, the effect of amylase copy numbers seems to depend on lifestyle,

36 and the mechanism of this effect was partially explained by changes in the oral and gut 
37 microbiome compositions; however, a detailed mechanism has been unclarified. In this

38 study, we showed their association with diabetes in addition to obesity, and further

39 discovered a plausible mechanism of this association based on the function of

40 commensal bacterial in a northern Japanese population. First, we confirmed that the

41 amylase copy number in the population tends to be larger than that reported in other

42 studies and that there is a positive association between obesity and diabetes ( $\mathrm{p}=1.95 \mathrm{E}-2$

43 and 3.28E-2). Second, we identified that relative abundance of some genus level

44 microbiome, Capnocytophaga, Dialister, and previously reported bacteria, were

45 significantly associated with amylase copy numbers. Finally, through functional

46 gene-set analysis using shotgun sequencing, we observed that the abundance of genes in

47 the Acarbose pathway in the gut microbiome was significantly decreased with an

48 increase in the amylase copy number ( $\mathrm{p}$-value $=5.80 \mathrm{E}-4)$, which can partly explain the

49 mechanism underlying obesity and diabetes in populations with high amylase copy

50 numbers.

51

\section{Keywords}

53 amylase copy number, whole-genome sequencing, shotgun metagenomic sequencing,

54 obesity, diabetes, acarbose pathway

\section{Introduction}

58 Similar to hundreds of human genes, the amylase genes reside in a structurally complex

59 locus, with inversions, deletions, and many duplications. Copy number variation (CNV)

60 of AMY genes, which encode amylase enzymes that digest starch into maltose and

61 dextrin, is considered one of the strongest signals of recent natural selection in the

62 human population. There are three amylase genes; the copy number of AMY1

63 (AMY1-CN), which encodes salivary amylase, varies from 1 to more than 20 copies,

64 whereas the copy numbers of AMY2A (AMY2A-CN) and AMY2B (AMY2B-CN),

65 which encode pancreatic amylase, vary from 1 to more than 6 copies. AMY1-CN is

66 known to be positively correlated with salivary amylase activity and hydrolyzes the

67 alpha bonds of starch and glycogen, initiating the process of starch degradation in the

68 mouth. As the variety in the number of these genes is specific to agricultural races

69 among humans, the CNVs of these three genes are hypothesized to shape the metabolic

70 response to dietary starch (1-3). 
72 In recent decades, several studies have shown that AMY1-CN and salivary amylase

73 concentrations are associated with obesity and diabetes. Further, an increase in

74 AMY1-CN levels is reported to decrease the risk of obesity (4-9). However, some other

75 studies have reported conflicting results and showed no or contrasting associations of

76 AMY1-CN with obesity (10-12). These conflicting results may be due to the

77 heterogeneity in patient samples in terms of genetic background and lifestyle, as well as

78 the use of different study methods, such as the assessment of AMY1-CN. Interestingly,

79 Rukh et al. reported that the degree of dietary starch intake modifies the relationship

80 between copy number variation in the salivary amylase gene and BMI; thus, an increase

81 in AMY1-CN decreases the risk of obesity in the low starch intake group and increases

82 the risk in the high starch intake group (12). Marcovecchio et al. also showed that an

83 increase in AMY1-CN decreases the risk of obesity in boys, but can increase its risk in

84 girls (9). Some other studies have shown that even though AMY1-CN is associated with

85 salivary amylase levels, the salivary amylase level is more associated with obesity and

86 BMI compared to AMY1-CN (13-15).

87

88 Humans with high AMY1-CN (AMY1H), who can produce high levels of salivary

89 amylase, might thus derive more energy from the same amount of diet compared to

90 those with low AMY1-CN (AMY1L). Compared to AMY1H, AMY1L might be

91 expected to harbor gut microbiomes with a greater capacity for the decomposition of

92 carbohydrates. Thus, AMY1 copy number has also recently emerged as a significant

93 factor in shaping the relative abundance of the specific members in the human oral and

94 gut microbiota (16-18). Further, an experimental study elucidated that the types of

95 microbes in AMY1H drive higher adiposity when transferred to germ-free mice (18),

96 but the detailed mechanism has not been clarified.

97

98 In this study, we aimed to investigate the fundamental relationships of AMY1 and

99 AMY2A with diabetes and obesity in a homogenous northern Japanese population

100 considered to have a high starch intake. We first identified AMY1-CN, AMY2A-CN,

101 and AMY2B-CN, and their mutation distributions using whole-genome sequencing data.

102 In many studies, CNV arrays have been used to detect CNVs; however, recent studies

103 suggest that whole-genome sequencing (WGS) analysis of CNVs is likely to be more

104 efficient and outperforms array-based CNV analysis (19). We utilized a methodology

105 that has been developed to accurately estimate CNVs in AMY regions from sequencing

106 data (20,21). Secondly, we analyzed the association of the copy numbers of AMY

107 genes (AMY-CNs) with obesity and diabetes. We then analyzed the oral and gut 
medRxiv preprint doi: https://doi.org/10.1101/2021.10.02.21264452; this version posted October 4, 2021. The copyright holder for this preprint (which was not certified by peer review) is the author/funder, who has granted medRxiv a license to display the preprint in perpetuity.

It is made available under a CC-BY-NC-ND 4.0 International license .

108 microbiome at the genus level to discriminate high and low AMY-CN individuals using

109

110

111

112

113

114

115

116

117

118

119

120

121

122

123

124

125

126

127

128

129

130

131

132

133

134

135

136

137

138

139

140

141

142

143

$16 \mathrm{~S}$ rRNA gene sequence analysis. Finally, we obtained shotgun whole-genome

sequencing data of the oral and gut microbiome to investigate the functional gene sets

of starch degradation pathways that can be related to AMY-CNs.

\section{Results}

\section{Distribution of AMY1, AMY2A, and AMY2B copy number variation}

We first analyzed the WGS data to detect AMY-CN from 1,479 individuals in the 2015 and 2016 participants. For estimating AMY-CN, we applied TIDDIT (20) and AMYCNE (21), and then obtained AMY1A, AMY2A, and AMY2B CNs and their relationships, as illustrated in Fig. 1. In contrast to the average AMY1-CN 10.3 in this population, as described in Fig. 1(a), previous studies have reported much smaller average AMY1-CNs; for example, the average CN was 7 in a study from Qatar (13), 8.3 in a study from Japan (14), 7.9 in a study from the US (18), and 7.0 in a study from Mexico (16). As illustrated in Fig. 1(b), AMY1 and AMY2A CNs were strongly correlated $(r=0.60, p=2.82 \mathrm{E}-144)$ because their genetic regions are near and on the same linkage disequilibrium (LD) block (11). On the contrary, AMY2B-CN was not associated, and almost all participants had two copies. Thus, we focused on AMY1 and AMY2A CNs in the following experiments.

\section{SNP patterns on AMY1, AMY2A and AMY2B regions}

Next, we summarized the SNP distributions in the AMY gene regions as shown in Table 1 and illustrated in Fig. 2. Contrary to the results of AMY CNV, the number of SNPs was clearly larger in the AMY2A and AMY2B regions than in the AMY1 region. However, a previous study reported that several SNPs with a minor allele frequency of more than 0.3, for example, rs42443712, were detected in the AMY1A region (11). In addition, several SNPs discovered in our analysis did not have a reference SNP ID (rsID) assigned to an identical location. It could be that the genetic variety of AMY1A might not be observed because this cohort is from a region of Japan where genetic diversity appears to be low. Notably, even though the effects of these SNPs on BMI and HbA1c were investigated, no significant effect was detected, similar to a previous study (11). Thus, we focused on the effects of AMY-CN in the following sections.

\section{Effect of AMY CN on HbAlc and BMI}

Then, we performed a linear regression analysis of AMY1 and AMY2A CNs on HbA1c and BMI with the explained covariates. Therefore, AMY1-CNs and AMY2-CNs were 
144 positively associated with $\mathrm{HbA1c}$ and BMI, and their p-values for the first and second

145 criteria of AMY1-CN and AMY2A-CN were 1.34E-2, 1.95E-2, and 2.76E-2 for HbA1c

146 and 4.71E-1, 3.28E-2, and 6.25E-2 for BMI, respectively, as illustrated in Fig3. Using

147 the predefined threshold $(\mathrm{p}=0.05)$, we confirmed the statistically significant association

148 of AMY1 and AMY2A CNs with HbA1c. However, only AMY1A in the second

149 criterion tended to be associated with BMI. These results are partially consistent with

150 those of a previous study (12); further, the association with HbA1c in the Japanese

151 population seems to be a novel observation.

152

\section{Association analysis between genus level microbiome composition and AMY CNS}

154 In this study, we obtained the oral and gut microbiome composition of the participants in 2015, 2016, 2017, and 2018 through 16S rRNA sequencing. Among these, we extracted the data from participants whose WGS was also obtained to calculate AMY-CNs in 2014 or 2015. Considering the exclusion criteria, we selected 451, 487, 426, and 483 participants for the oral microbiome and 435, 475, 412, and 465 participants for gut microbiome analysis in 2015, 2016, 2017, and 2018, respectively. In the microbiome analysis, we excluded bacteria for which the sum of compositions of all participants was less than 1.0E-5 and (ii) those that were included in less than $3 \%$ of the participants each year. We thus retained 90 and 135 genus level bacteria for the oral and gut microbiome analyses, respectively. Thus, their predefined significant level with Bonferroni correction were $0.05 / 90=5.56 \mathrm{E}-4$ and $0.05 / 135=3.70 \mathrm{E}-4$, respectively. Because it was difficult to integrate microbiome composition data over the entire study period, we calculated integrated p-values using fixed-effect meta-analysis based on the estimates and standard errors. The obtained results are listed in Table 2; we extracted two prominent bacteria associated with AMY1-CNs, as illustrated in Fig.4. Capnocytophaga in the oral microbiome tended to be negatively associated with AMY1-CN ( $\mathrm{p}=1.38 \mathrm{E}-6)$. In addition, the Dialister composition in the gut microbiome increased and decreased with the increase in AMY1 CNs for males and females, respectively, and these differences were statistically significant $(\mathrm{p}=1.02 \mathrm{E}-6$ for males and 6.00E-6 for females). In particular, Dialister has been reported to be associated with carbohydrate metabolism and host diet; we could reproduce these results $(18,22)$. The top 10 bacteria and their p-values are presented in the supplementary material.

176

\section{Functional gene abundance analysis}

178 Through the above experiments, AMY-CNs can be associated with HbA1c levels, BMI, 179 and the oral and gut microbiomes. To further investigate the underlying mechanisms, we 180 analyzed the abundance of functional gene sets in the oral and gut microbiome in 
181 association with AMY-CNs using shotgun microbiome sequencing. As we obtained data

182 for 2015 and 2016, we considered participants whose WGS was obtained in 2014 and

183 2015. In this experiment, we generated the data by selecting the participants in 2015 and

184 those participants in 2016 who did not participate in 2015, and integrating them into a

185 single table. A total of 617 participants were thus included in this experiment. Because

186 AMY-CNs determine the capacity of amylase secretion, which decomposes starch into

187 dextrin and maltose, we focused on the starch degradation pathway. In the KEGG

188

189

190

191

192

193

194

195

196

197

198 pathway database (23), we selected starch and sucrose metabolism (KEGG Identifier: map00500), amino sugar and nucleotide sugar metabolism (map00520), and Acarbose and validamycin biosynthesis (map00525) as potentially associated pathways. For this analysis, the total abundance of the gene set in each pathway was calculated for each participant, and a linear regression analysis with predefined covariates was also performed to analyze the association with AMY-CNs. Here, the predefined p-value with Bonferroni correction was $0.05 / 3=1.67 \mathrm{E}-2$. As a result, the total abundance of genes in map0525 was negatively associated with AMY1-CN under the first and second criteria (p $=5.80 \mathrm{E}-4$ and 3.97E-2, respectively) and with AMY2A-CN (p-value $=1.15 \mathrm{E}-3$ ), as illustrated in Fig.5. Acarbose is an oral hypoglycemic drug used for treating type 2 diabetes, and this decrease could increase blood glucose levels.

199

\section{Discussion and Conclusion}

201 Several studies published in the last decade have shown that AMY1-CN is associated

202 with obesity and diabetes. In contrast, several studies have reported that no significant

203 association was observed among them. These conflicting results may have been

204 influenced by the selection of populations and their lifestyles. For example, in people

205 who eat a low starch diet, a high percentage of salivary amylase digests starch quickly

206 and serum glucose levels become quickly and temporarily high at an early stage,

207 whereas a low percentage of amylase can maintain a high serum glucose level. On the

208 contrary, for people who eat large amounts of starch, a high percentage of salivary

209 amylase may result in high glucose levels, whereas a low salivary amylase level may

210 maintain moderate serum glucose levels. For most human beings, grains and rice are a

211 major source of energy. Thus, it is important to elucidate the impact and mechanism of

212 AMY-CNV on obesity and diabetes.

213

214 First, we assumed that the residents of Hirosaki City consume a relatively large amount

215 of starch, even in Japan. According to a survey of rice consumption in two or more

216 households by the Statistics Bureau, Ministry of Internal Affairs and Communications

217 in Japan (https://www.stat.go.jp/data/kakei/5.html), the national average is 64.16

218 [kg/year], the consumption in Morioka city, which is close to Hirosaki city, is 71.61

219 [kg/year], whereas that in Tokyo is 53.16 [kg/year]; thus, the high starch consumption

220 in this area might be related to this observation. Further, the AMY1A region has very

221 few SNPs compared to the AMY2A and AMY2B regions. Thus, it seems that AMY1A 
medRxiv preprint doi: https://doi.org/10.1101/2021.10.02.21264452; this version posted October 4, 2021. The copyright holder for this preprint (which was not certified by peer review) is the author/funder, who has granted medRxiv a license to display the preprint in perpetuity.

It is made available under a CC-BY-NC-ND 4.0 International license .

222 exhibits diversity by copy number, and the AMY2A and AMY2B express diversity by

223 mutation. However, in previous studies, more frequent SNPs in the AMY1 region have

224 been reported; these results might thus be region-specific.

225

226 Similar to previous studies, we examined the association between AMY1-CN and

$227 \mathrm{HbA1c}$ and BMI. The results showed that both HbA1c levels and BMI increased

228 significantly with the increase in AMY1-CN. This is consistent with the finding of a

229 positive correlation between the increase in AMY1-CN and BMI in the high starch

230 intake group (12), but the association with HbA1c levels is a new finding in the

231 Japanese population. Further, because the average AMY1-CN in this study was clearly

232 greater than that in other studies, there could be a positive selection that favored more

233 AMY1-CN in the past.

234

235 AMY-CN is reported to have a strong correlation with amylase secretion, and has a

236 stronger effect on BMI and the oral and gut microbiome. As the amount of amylase

237 secretion was not measured in this study, we examined the association between

238 AMY1-CN and the oral and gut microbiome. Previously, the association of the human

239 microbiome and AMY-CNs and the effect of microbiome translocation with obesity

240 have been reported (16-18). Those studies have reported that Prevotella in the oral

241 cavity and Ruminococcus in the gut increased with an increase in AMY1-CN. In our

242 experiment, although not statistically significant, similar tendencies were observed. In

243 particular, in this study, we found that Capnocytophaga decreased significantly in the

244 oral cavity as AMY1-CN increased. Capnocytophaga is known to be associated with

245 periodontal disease and could be affected by the amount of starch decomposition;

246 however, to the best of our knowledge, no study has been published regarding its

247 associations. In addition, Dialister increased significantly in men and decreased

248 significantly in women with an increase in AMY1-CN. Dialister has been reported to

249 increase in obese women, but we show a strong correlation with AMY1-CN rather than

250 with BMI and HbA1c levels. The amount of Dialister may be thus directly affected by

251 the amount of amylase secreted, which could result in obesity and other consequences.

252

253 Finally, we tried to elucidate the mechanism underlying the effect of the oral and gut

254 microbiome on obesity and diabetes from the viewpoint of changes in the abundance of

255 pathway-based functional genesets. The results showed that the total amount of

256 acarbose and validamycin biosynthesis (map00525) decreased significantly with an

257 increase in both AMY1-CN and AMY2A-CN. Acarbose is an oral hypoglycemic drug 
258 used for the treatment of type 2 diabetes, which delays starch breakdown to glucose by 259 inhibiting the action of $\alpha$-glucosidase. Thus, glucose is absorbed more slowly into the

260 body, and postprandial hyperglycemia can be suppressed. In other words, in the group

261 with a small number of AMY-CN, the expression of acarbose in the gut can be

262 increased and the increase in blood glucose is suppressed; in contrast, with a large

263 number of AMY-CN, the expression of acarbose is decreased and the increase in blood

264 glucose is promoted. Through this mechanism, we could partially explain the causes of

265 an increase in $\mathrm{HbA} 1 \mathrm{c}$ levels and BMI in the group with a large AMY-CN.

266

267 Within this study, we considered a particularly limited population in Japan, and so,

268 these results may be specific to lifestyle, genome, and the oral and gut microbiome, and

269 may not be applicable to other populations. However, even in such a specific population,

270 our findings are partially consistent with previous research, and novel findings on

271 AMY-CN have been further obtained.

272

273 Materials and Methods

274 Ethics statement

275 This study design was approved by the Hirosaki University Hospital Institutional

276 Review Board (Protocol Number Num. 2017-026). Written informed consent was

277 obtained from all the patients in this study. No animal experiments were conducted in

278 this study.

279

280

281

282

283

284

285

286

287

288

289

290

291

292

293

294

\section{Sample collection and preparation}

We analyzed the data obtained from the Hirosaki Center of Innovation (COI) health promotion project, which has collected cohort data from the year 2005 in Hirosaki (the population is approximately 10,000) in northern Japan. Each year, approximately 1,000 people have their health status checked through a free participation program. The dataset actually has numerous missing data because the participants do not necessarily undergo a checkup each year. Thus, the number of analyzed participants was displayed in each experiment. Questionnaires and blood tests were collected over the entire study period, and single nucleotide polymorphisms (SNPs) were identified for participants in 2014, 2015, 2016, and 2017; WGS analysis was performed for participants in 2014 and 2015; 16S rRNA sequencing of the oral and gut microbiomes was performed for participants in 2015, 2016, 2017, and 2018, and whole-genome shotgun sequencing of the oral and gut microbiomes was performed for participants in 2015 and 2016.

295 The main exclusion criteria were as follows: (1) participants were younger than 18 years

296 of age or were 55 older; (2) those with an estimated glomerular filtration rate (eGFR) 
297 below $60\left[\mathrm{~mL} / \mathrm{min} / 1.73 \mathrm{~m}^{2}\right]$ as calculated from serum creatinine levels and age at

298 admission; (3) those with serum total bilirubin levels above 2 [mg/dl], (4) those with

299 serum albumin levels above 35 [g/dl], (5) those with a BMI above 35 or below 18, (6)

300 those who were prescribed medicine for diabetes mellitus as per their questionnaires, (7)

301 those who were prescribed antidiabetic or antibiotic medication as per their

302 questionnaires, for the analysis of oral and gut microbiome; and (8) those with missing

303 information regarding any of their covariates.

304

305 The participant covariate information was obtained from the blood value tests and

306 questionnaires. The questionnaires were administered upon induction into the study. (a)

307 Age and (b) sex information were obtained from questionnaires (sex was also checked

308 using genetic data), (c) smoking status was classified as never (0) and current and former

309 (1); (d) drinking status was classified as non-drinker (0), and current and former drinker

310 (1). Participants who used medication for (e) high blood pressure, (f) dyslipidemia, (g)

311 steroids, and (h) antibiotics were flagged as non-use (0) and use (1). Finally, we

312 performed principal component analysis (PCA) for genotyping using the algorithm

313 implemented in smartpca (24), and used the top five principal components (PCs) as

314 covariates to represent the population structure based on the genetic correlations among

315 individuals.

316

317 SNPs and whole genome sequencing

318 DNA was purified from peripheral whole blood using a DNA extraction kit (QIAamp

31996 DNA Blood Kit (QIAGEN, Hilden, Germany)) and was extracted from plasma

320 pellets for SNP arrays and whole genome sequencing, respectively. SNP information

321 was obtained from Japonica Array (Toshiba, Tokyo, Japan) (25) and comprised

322 population-specific SNP markers designed from the 1070 whole genome reference

323 panel (26), and whole genome sequencing was performed by Takara Bio Corporation

324 (Shiga, Japan).

325

326 Analysis of AMY1, AMY2A, and AMY2B copy number variations

327 To detect AMY1, AMY2A, and AMY2B CNVs from the WGS data, we used

328 AMYCNE (21), which was developed to estimate the AMY CNV from sequencing data.

329 The input data for this software was prepared using TIDIT (20) which was also

330 developed to clarify the structural variants.

331

332

SNP analysis for AMY1, AMY2A, and AMY2B regions 
333 For SNP detection, we applied the Genomon pipeline

334 (https://github.com/Genomon-Project), which has been developed to investigate

335 mutations from exome/whole genome sequencing data. The filter conditions were as

336 follows: minor allele frequency of 0.02 or higher, minimum read depth higher than 8 ,

337 and $10 \%$ posterior percentile of the minor allele count based on a binomial distribution

338 greater than 0.02 , and a minimum base quality of 15 or higher.

339

340

\section{Microbiome assay}

341 Microbiome samples were obtained from participants' tongue plaques and stool samples

342 for oral and gut microbiomes, respectively, and were stored at $-80^{\circ} \mathrm{C}$ until use. The

343 detailed library preparation method, including PCR conditions, has been described in a

344 previous paper (27). Briefly, the samples were mixed with zirconia beads and lysed using

345 a FastPrep FP100A instrument (MP Biomedicals, Santa Ana, California, USA). DNA

346 was extracted from the bead-treated suspensions using a Magtration System 12GC and

347 GC series MagDEA DNA 200 (Precision System Science, Chiba, Japan) and amplified.

348 The 16S rRNA gene amplicons covering the V3-V4 region were sequenced using

349 Pro341F and Pro805R primers. Sequencing was performed using a paired-end,

$3502 \times 250$-base pair cycle run on an Illumina MiSeq sequencing system. Shotgun whole

351 genome sequencing was performed on the HiSeq2500 instrument (Illumina, San Diego,

352 California, USA) with $101 \square$ bp paired-end reads.

353

354 Calculation of genus level microbiome abundance profiles from 16S rRNA sequences

355 Adapter sequences and low-quality bases at the $3^{\prime}$-end (threshold $=30$ ) were trimmed

356 using Cutadapt (version: 1.13) (28) from paired-end reads obtained using the Illumina

357 MiSeq sequencing system. In addition, the reads containing $\mathrm{N}$ bases and reads shorter

358 than 150 bases were removed. The remaining reads were merged into single reads, and

359 those with a length of 370 to 470 bases were removed using the VSEARCH (29)

360 fastq_mergepairs subcommand (version: version 2.4.3). After merging, we removed

361 reads that were expected to have one or more base errors from the base quality. After

362 removing chimeric reads using the VSEARCH (29) uchime_denovo subcommand, the

363 reads were clustered with $97 \%$ sequence identity and the taxa of each cluster were

364 identified using the RDP Classifier (git commit hash: (commit hash:

365 701e229dde7cbe53d4261301e23459d91615999d)) (30). At this time, the result with a

366 confidence value $<0.8$, was treated as unclassified. The relative abundance of each

367 taxon was then calculated by dividing the number of reads belonging to the cluster to

368 which the taxon was assigned by the total number of reads. 
medRxiv preprint doi: https://doi.org/10.1101/2021.10.02.21264452; this version posted October 4, 2021. The copyright holder for this preprint (which was not certified by peer review) is the author/funder, who has granted medRxiv a license to display the preprint in perpetuity.

It is made available under a CC-BY-NC-ND 4.0 International license .

Calculation of functional gene abundance from shotgun metagenomic sequencing

371 Adapter sequence and low-quality bases at the 3 '-end (threshold $=30$ ) were trimmed

372 using Cutadapt (version: 1.13) from $2 \times 100$-base paired-end reads obtained using the

373 Illumina HiSeq 2500 system. Reads containing N bases and reads shorter than $80 \mathrm{bp}$

374 were removed. Reads from the host genome were also removed using BWA-MEM

375 (version 0.7.15) (31) with GRCh38. From the remaining reads (referred to as non-host

376 reads), contigs were generated by metagenomic assembly using MEGAHIT (version

377 1.1.1) (32). The coding regions of genes on these contigs were predicted using Prodigal

378 (version 2.6.3) (33). After mapping the non-host reads to contigs using BWA-MEM, the

379 relative abundances of the genes were calculated from the average depth of the gene

380 region. KEGG pathway annotations were mapped to genes using a sequence similarity

381 search with MMseqs2 (version: 1d2579627f43662ecaaa0778bd348fc35048976a) (34).

382 We used 1E-10 as the E-value threshold for the similarity search.

383

384 Statistical Analysis

385 In the first criterion for AMY1-CN, participants were divided into low (AMY1-CN

386 range 3-10) and high (AMY1-CN range 11-24) groups, which were separated at the

387 center line. In the second criterion for AMY1-CN, participants were divided into the

388 low (AMY1-CN range, 3-7), medium (range 8-13), and high (range, 14-24) groups.

389 The minimum copy number above 10 percentile and the maximum copy number below

39090 percentile of AMY1-CN distribution was 7 and 14, respectively. Similarly, for

391 analyzing AMY2A-CN, participants were divided into low (AMY2A-CN range 1-2)

392 and high (AMY2A-CN range 3-5) groups at the center line. We could not analyze the

393 effect of AMY2B-CN because almost all patients had two AMY2B-CNs. For these

394 groups, linear regression analysis using the above-explained covariates was applied to

395 detect statistical significance. A p-value less than 0.05 was considered to indicate

396 statistical significance when considering the corrections for multiple tests.

397

398 Acknowledgments

399 This work was supported by JST COI Grant Number JPMJCE1302. This research used

400 the supercomputing resources provided by the Human Genome Center, Institute of

401 Medical Science, University of Tokyo (http://sc.hgc.jp/shirokane.html).

402

403 Declaration of interests

404 The authors declare no competing financial interests. 


\section{Authors' contributions}

407 T.H. designed the study, analyzed the data, interpreted the data, and wrote the

408 manuscript. M. K., R.Y., and N.S. analyzed the data, interpreted the data, helped write

409 the manuscript, and commented on the drafts. M.A., J.S., I.T., and K.M. performed the

410 medical checkups and gathered and summarized the data. S.I. interpreted the data,

411 helped write the manuscript, commented on the drafts, and supervised the study. All

412 authors have read and approved the final manuscript.

413

414 Data Availability

415 The datasets supporting the current study have not been deposited in a public repository

416 [because the data are not public] but are available from the corresponding author, S.I.,

417 on request.

418

419 References

420

421 1. Pajic P, Pavlidis P, Dean K, Neznanova L, Romano RA, Garneau D, et al. Independent amylase 422 gene copy number bursts correlate with dietary preferences in mammals. Elife. 2019;8.

423 2. Perry GH, Dominy NJ, Claw KG, Lee AS, Fiegler H, Redon R, et al. Diet and the evolution of

424 human amylase gene copy number variation. Nat Genet. 2007;39(10):1256-60.

425 3. Inchley CE, Larbey CD, Shwan NA, Pagani L, Saag L, Antao T, et al. Selective sweep on 426 human amylase genes postdates the split with Neanderthals. Sci Rep. 2016;6:37198.

427 4. Falchi M, El-Sayed Moustafa JS, Takousis P, Pesce F, Bonnefond A, Andersson-Assarsson JC, 428 et al. Low copy number of the salivary amylase gene predisposes to obesity. Nat Genet. 429 2014;46(5):492-7.

430 5. Mejia-Benitez MA, Bonnefond A, Yengo L, Huyvaert M, Dechaume A, Peralta-Romero J, et al.

431 Beneficial effect of a high number of copies of salivary amylase AMY1 gene on obesity risk in Mexican 432 children. Diabetologia. 2015;58(2):290-4.

433 6. Bonnefond A, Yengo L, Dechaume A, Canouil M, Castelain M, Roger E, et al. Relationship 434 between salivary/pancreatic amylase and body mass index: a systems biology approach. BMC Med. $4352017 ; 15(1): 37$.

436 7. Elder PJD, Ramsden DB, Burnett D, Weickert MO, Barber TM. Human amylase gene copy 437 number variation as a determinant of metabolic state. Expert Rev Endocrinol Metab. 2018;13(4):193-205.

438 8. Viljakainen H, Andersson-Assarsson JC, Armenio M, Pekkinen M, Pettersson M, Valta H, et al. 439 Low Copy Number of the AMY1 Locus Is Associated with Early-Onset Female Obesity in Finland. PLoS 440 One. 2015;10(7):e0131883. 
4419 9. Marcovecchio ML, Florio R, Verginelli F, De Lellis L, Capelli C, Verzilli D, et al. Low AMY1

442 Gene Copy Number Is Associated with Increased Body Mass Index in Prepubertal Boys. PLoS One.

443 2016;11(5):e0154961.

444 10. Yong RY, Mustaffa SB, Wasan PS, Sheng L, Marshall CR, Scherer SW, et al. Complex Copy

445 Number Variation of AMY1 does not Associate with Obesity in two East Asian Cohorts. Hum Mutat.

$4462016 ; 37(7): 669-78$.

447 11. Usher CL, Handsaker RE, Esko T, Tuke MA, Weedon MN, Hastie AR, et al. Structural forms

448 of the human amylase locus and their relationships to SNPs, haplotypes and obesity. Nat Genet.

449 2015;47(8):921-5.

450 12. Rukh G, Ericson U, Andersson-Assarsson J, Orho-Melander M, Sonestedt E. Dietary starch 451 intake modifies the relation between copy number variation in the salivary amylase gene and BMI. Am J 452 Clin Nutr. 2017;106(1):256-62.

453 13. Al-Akl N, Thompson RI, Arredouani A. High plasma salivary alpha-amylase, but not high 454 AMY1 copy number, associated with low obesity rate in Qatari adults: cross-sectional study. Sci Rep. $4552020 ; 10(1): 17918$.

456 14. Higuchi R, Iwane T, Iida A, Nakajima K. Copy Number Variation of the Salivary Amylase 457 Gene and Glucose Metabolism in Healthy Young Japanese Women. J Clin Med Res. 2020;12(3):184-9.

458 15. Nakajima K, Higuchi R, Iwane T, Iida A. The association of low serum salivary and pancreatic 459 amylases with the increased use of lipids as an energy source in non-obese healthy women. BMC Res 460 Notes. 2020;13(1):237.

461 16. Leon-Mimila P, Villamil-Ramirez H, Lopez-Contreras BE, Moran-Ramos S, Macias-Kauffer 462 LR, Acuna-Alonzo V, et al. Low Salivary Amylase Gene (AMY1) Copy Number Is Associated with 463 Obesity and Gut Prevotella Abundance in Mexican Children and Adults. Nutrients. 2018;10(11).

464 17. Hjorth MF, Christensen L, Larsen TM, Roager HM, Krych L, Kot W, et al. Pretreatment 465 Prevotella-to-Bacteroides ratio and salivary amylase gene copy number as prognostic markers for dietary 466 weight loss. Am J Clin Nutr. 2020;111(5):1079-86.

467 18. Poole AC, Goodrich JK, Youngblut ND, Luque GG, Ruaud A, Sutter JL, et al. Human Salivary 468 Amylase Gene Copy Number Impacts Oral and Gut Microbiomes. Cell Host Microbe. 2019;25(4):553-64 469 e7.

470 19. Zhou B, Ho SS, Zhang X, Pattni R, Haraksingh RR, Urban AE. Whole-genome sequencing 471 analysis of CNV using low-coverage and paired-end strategies is efficient and outperforms array-based 472 CNV analysis. J Med Genet. 2018;55(11):735-43.

473 20. Eisfeldt J, Vezzi F, Olason P, Nilsson D, Lindstrand A. TIDDIT, an efficient and comprehensive 474 structural variant caller for massive parallel sequencing data. F1000Res. 2017;6:664.

475 21. Eisfeldt J, Nilsson D, Andersson-Assarsson JC, Lindstrand A. AMYCNE: Confident copy 476 number assessment using whole genome sequencing data. PLoS One. 2018;13(3):e0189710. 
medRxiv preprint doi: https://doi.org/10.1101/2021.10.02.21264452; this version posted October 4, 2021. The copyright holder for this preprint (which was not certified by peer review) is the author/funder, who has granted medRxiv a license to display the preprint in perpetuity.

It is made available under a CC-BY-NC-ND 4.0 International license .

477 22. Muniz Pedrogo DA, Jensen MD, Van Dyke CT, Murray JA, Woods JA, Chen J, et al. Gut

478 Microbial Carbohydrate Metabolism Hinders Weight Loss in Overweight Adults Undergoing Lifestyle

479 Intervention With a Volumetric Diet. Mayo Clin Proc. 2018;93(8):1104-10.

480 23. Kanehisa M, Goto S. KEGG: kyoto encyclopedia of genes and genomes. Nucleic Acids Res. $4812000 ; 28(1): 27-30$.

482 24. Price AL, Patterson NJ, Plenge RM, Weinblatt ME, Shadick NA, Reich D. Principal 483 components analysis corrects for stratification in genome-wide association studies. Nat Genet. 484 2006;38(8):904-9.

485 25. Kawai Y, Mimori T, Kojima K, Nariai N, Danjoh I, Saito R, et al. Japonica array: improved 486 genotype imputation by designing a population-specific SNP array with 1070 Japanese individuals. J 487 Hum Genet. 2015;60(10):581-7.

488 26. Nagasaki M, Yasuda J, Katsuoka F, Nariai N, Kojima K, Kawai Y, et al. Rare variant discovery 489 by deep whole-genome sequencing of 1,070 Japanese individuals. Nat Commun. 2015;6:8018.

490 27. Takahashi S, Tomita J, Nishioka K, Hisada T, Nishijima M. Development of a prokaryotic 491 universal primer for simultaneous analysis of Bacteria and Archaea using next-generation sequencing. 492 PLoS One. 2014;9(8):e105592.

493 28. MARTIN M. Cutadapt removes adapter sequences from high-throughput sequencing reads. 494 EMBnetjournal. 2011;17(1):10-2.

495 29. Rognes T, Flouri T, Nichols B, Quince C, Mahe F. VSEARCH: a versatile open source tool for 496 metagenomics. Peerj. 2016;4.

497 30. Lan YM, Wang Q, Cole JR, Rosen GL. Using the RDP Classifier to Predict Taxonomic Novelty 498 and Reduce the Search Space for Finding Novel Organisms. Plos One. 2012;7(3).

499 31. Li H. Aligning sequence reads, clone sequences and assembly contigs with BWA-MEM. 500 arXiv:13033997v2 2013.

501 32. Li DH, Liu CM, Luo RB, Sadakane K, Lam TW. MEGAHIT: an ultra-fast single-node solution 502 for large and complex metagenomics assembly via succinct de Bruijn graph. Bioinformatics. 503 2015;31(10):1674-6.

504 33. Hyatt D, Chen GL, LoCascio PF, Land ML, Larimer FW, Hauser LJ. Prodigal: prokaryotic 505 gene recognition and translation initiation site identification. Bmc Bioinformatics. 2010;11.

506 34. Steinegger M, Soding J. MMseqs2 enables sensitive protein sequence searching for the 507 analysis of massive data sets. Nat Biotechnol. 2017;35(11):1026-8.

508

509

510

511

512 
(a)
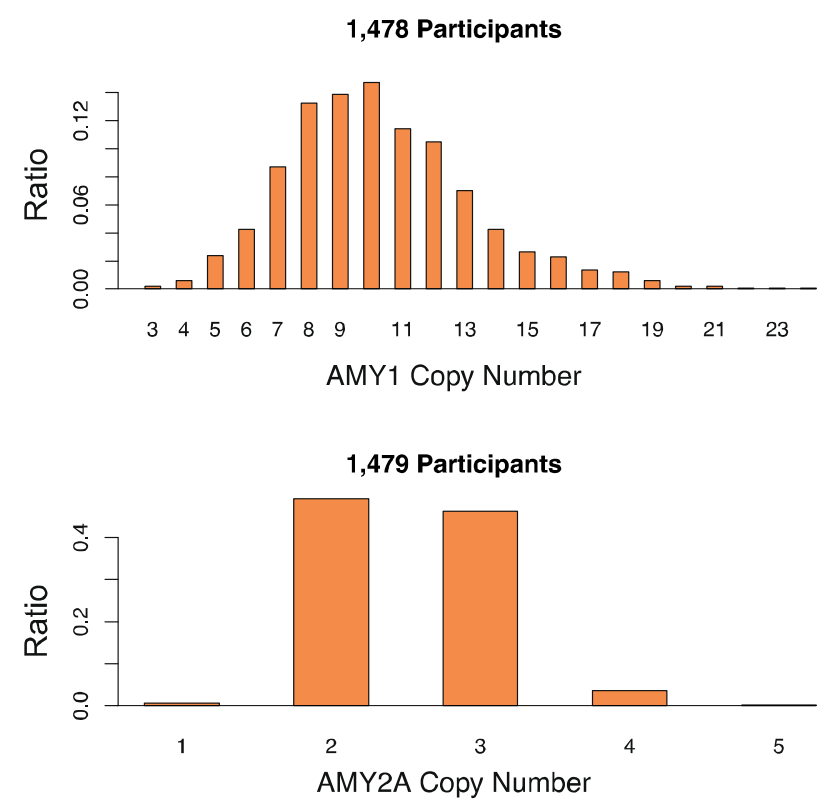

1,479 Participants

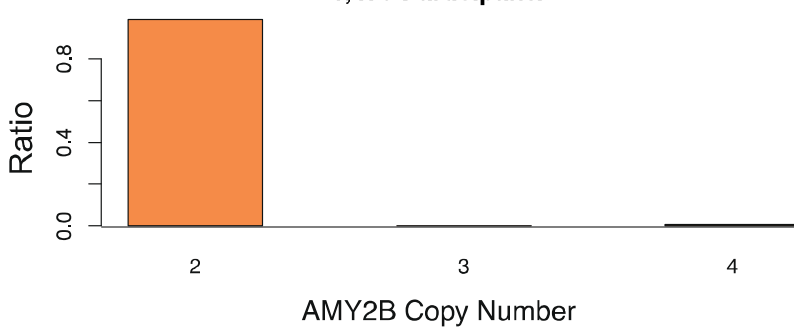

(b)
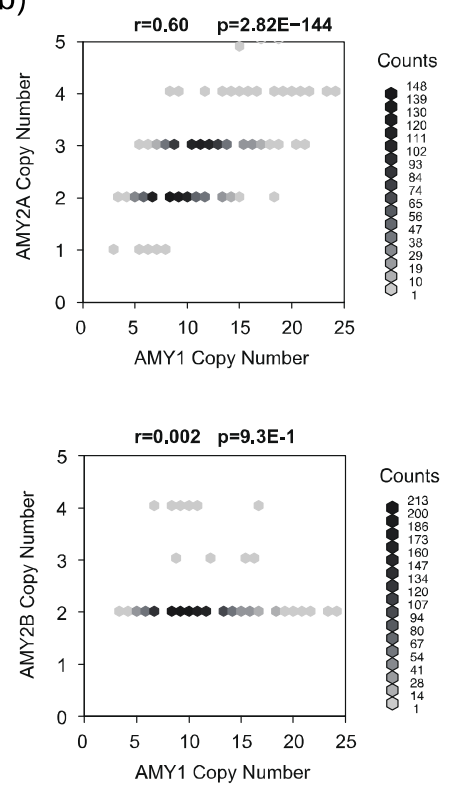

518

519 Figure 1. Histogram of AMY1, AMY2A, and AMY2B copy number variations and

520 their associations. In the left of this figure, the histograms of AMY1A, AMY2A, and

521 AMY2B-CNs are displayed. The number of analyzed participants are also indicated at

522 the top of each histogram. In the right of this figure, associations among AMY1A,

523 AMY2A, and AMY2B-CNs are illustrated as a scatter plot. At the top of each scatter

524 plot, $r$ and $p$ indicate Pearson's correlation coefficient and p-value, respectively. 


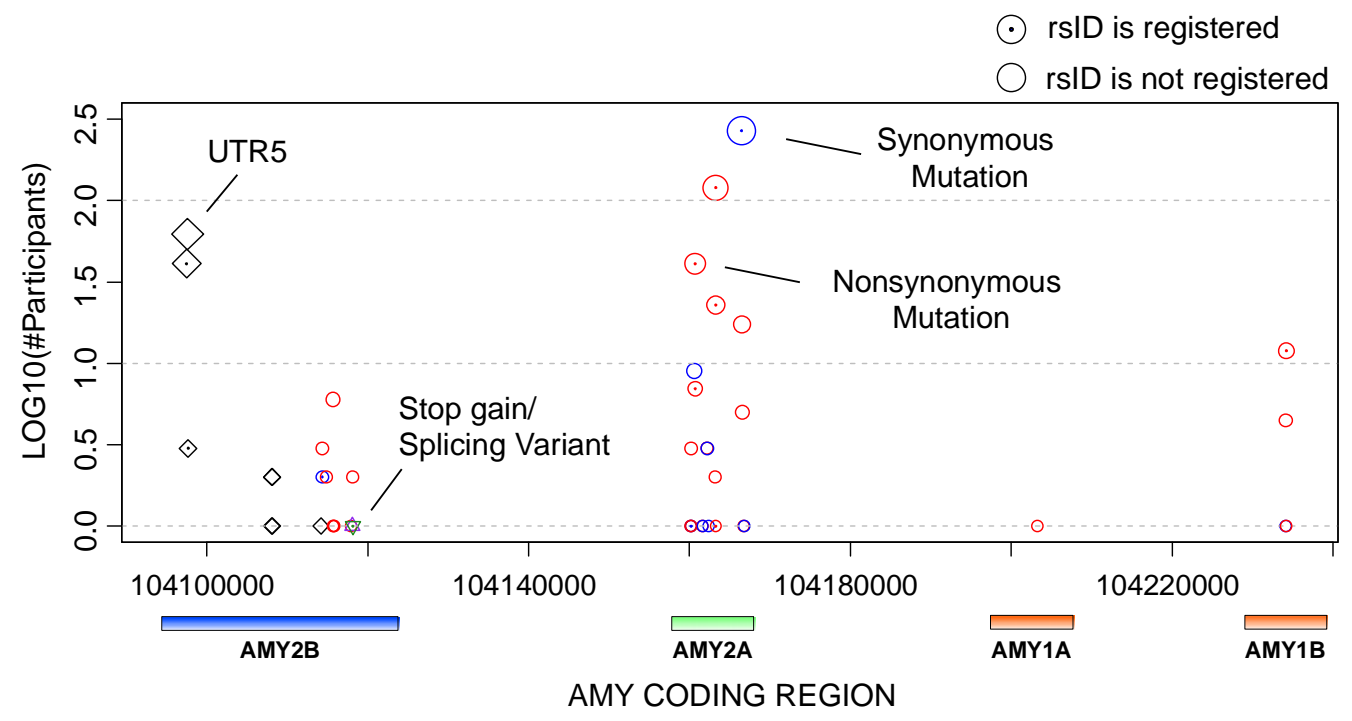

527 Figure2. Distributions of SNPs on the exonic regions of AMY1, AMY2A, and

528 AMY2B. In this figure, the vertical and horizontal axes indicate the number of

529 participants with the corresponding SNPs and genomic positions including AMY1A,

530 AMY2A, and AMY2B. Red, blue, green, black, and orange circles indicate

531 nonsynonymous, synonymous, stop-gain (nonsense), splicing site, and 5'

532 prime untranslated region mutations, respectively. The dot at the center of the circles

533 indicates that the SNPs have reference SNP IDs. Genes corresponding to the positions

534 are illustrated at the bottom of the figure. 

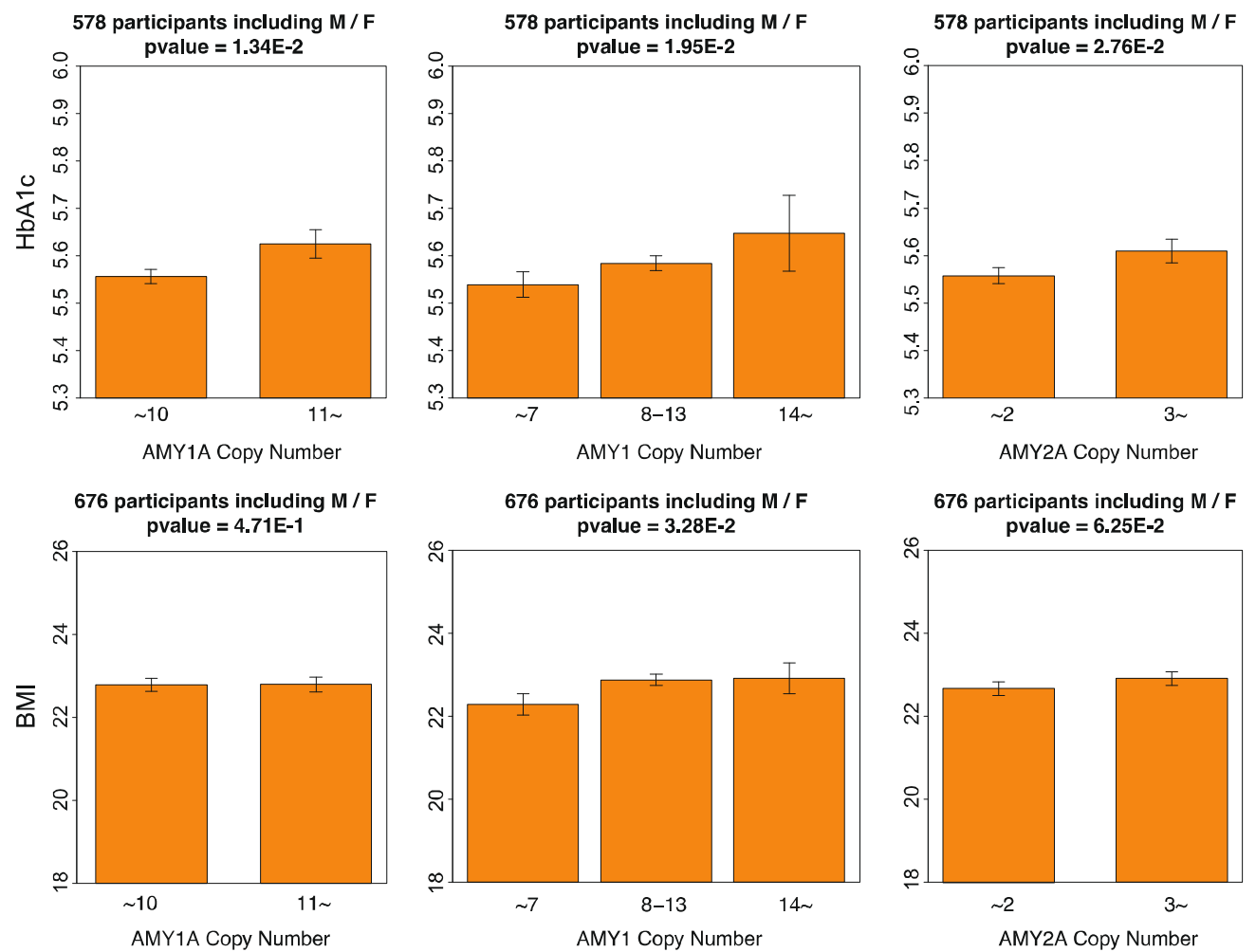

536 Figure3. Association of HbA1c and BMI with AMY1A and AMY2A-CNs. In the

537 upper and bottom parts of this figure, the associations of HbA1c and BMI with the

538 criterion 1 and 2 of AMY1-CNs and AMY2-CNs are illustrated as barplots, respectively.

539 The number of analyzed participants and p-values calculated using linear regression

540 models with predefined covariates are displayed on the barplots. 
(a)

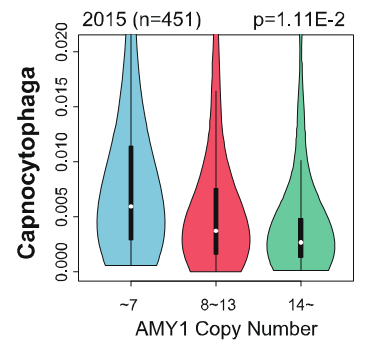

(b)
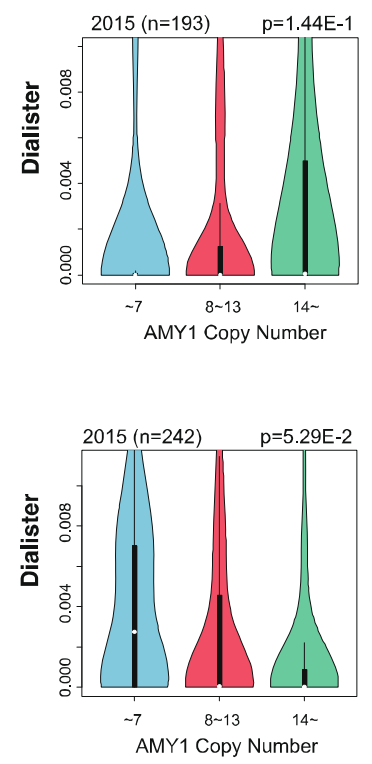

$16 S$ rRNA Bacterial Flora in Oral

Male / Female Participants (meta-p=1.38E-06)
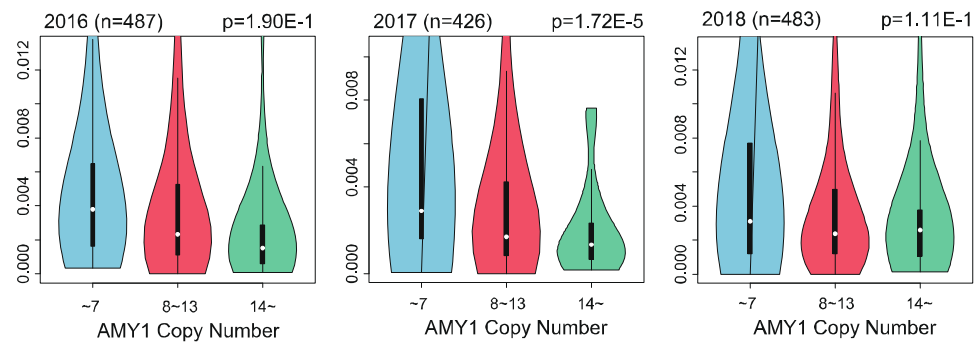

$16 S$ rRNA Bacterial Flora in Guts

Male Participants (meta-p=1.02E-06)
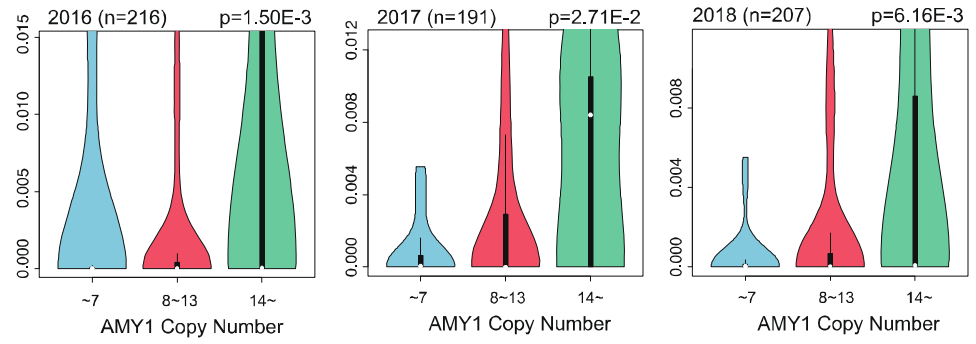

Female Participants (meta-p=6.00E-06)
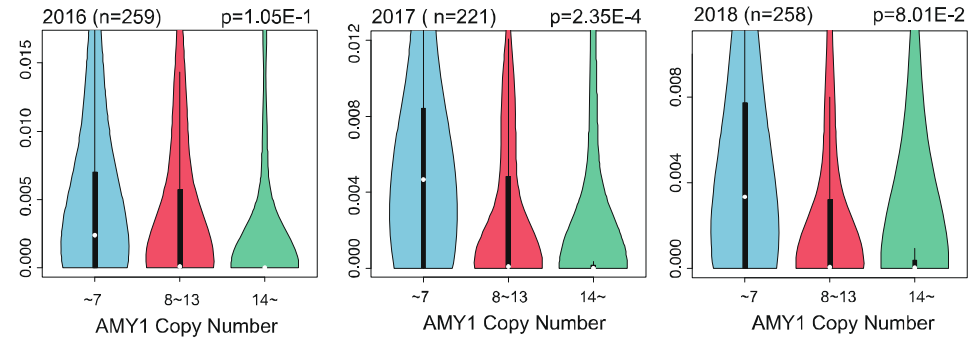

Figure4. Association of the oral and gut microbiome with AMY1-CN. In the upper,

544 middle, and bottom panels of this figure, associations of the composition of

545 Capnocytophaga for males and females, Dialister for males, and Dialister for females

546 are illustrated by violin-plot. The number of analyzed participants for each year and the

547 p-values calculated using linear regression models with predefined covariates are

548 displayed on the plots. 

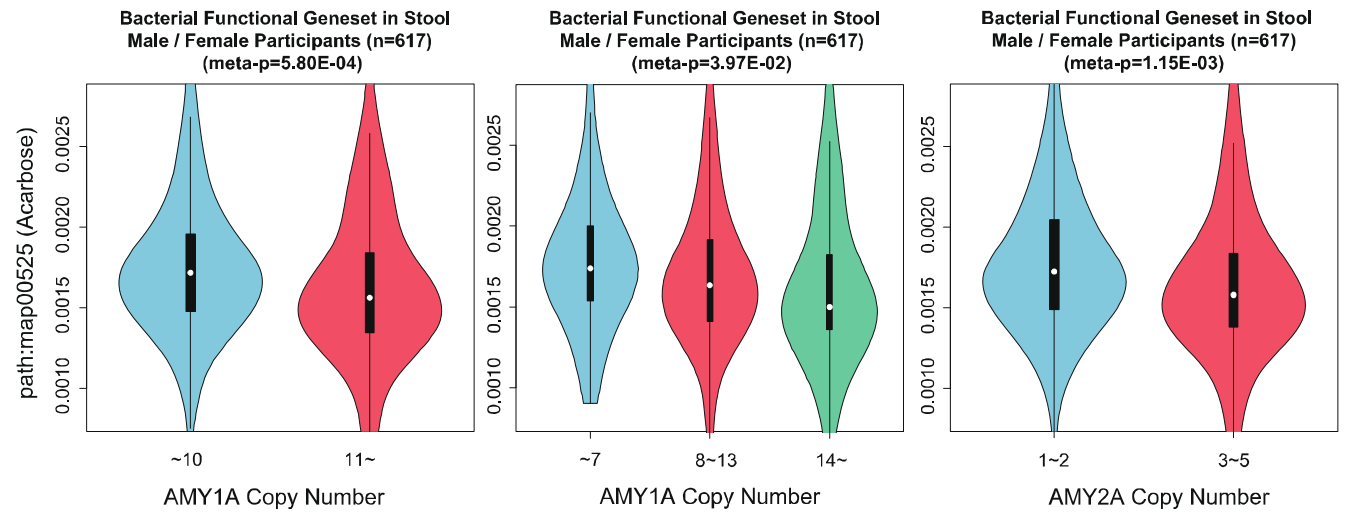

550 Figure 5. Associations of abundance of the Acarbose pathway gene set. The

551 associations of the total abundance of the gene-set in the Acarbose pathway (KEGG:

552 map00525) to criterion 1 and 2 of AMY1-CNs and AMY2-CNs are illustrated as

553 violin-plots. The number of analyzed participants and $\mathrm{p}$-values calculated by linear

554 regression models with predefined covariates are displayed on the plots.

555

556

Tables

557 Table 1. Summary of SNPs detected in the AMY1, AMY2A, and AMY2B gene

558 regions exonic (nonsynonymous, synonymous, top) intronic UTR5 splicing intergenic

AMY1 $20(19,1,0) \quad 1,131$ $564(251,292,1) \quad 13,253$ 13

583,736

AMY2

AMY1-AMY

2

Total 
medRxiv preprint doi: https://doi.org/10.1101/2021.10.02.21264452; this version posted October 4, 2021. The copyright holder for this preprint (which was not certified by peer review) is the author/funder, who has granted medRxiv a license to display the preprint in perpetuity.

\author{
It is made available under a CC-BY-NC-ND 4.0 International license.
}

\title{
570 Supplementary Material
}

\begin{tabular}{|c|c|c|c|c|c|c|c|c|c|c|c|}
\hline AMI & IYIA & AMY & IY2A & & IM1A & AMI & ILA & 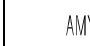 & (1) & & \\
\hline ocytophas & $1.38492 E-06$ & 6 Alloscardovia & 0.00252246 & Bacteroides & 0.00884817 & Mycoplasma & 0.00537596 & Capnocytoph & 1 9.7757E-07 & Howarcella & 0.00122281 \\
\hline Lautropia & 0.002875591 & 1 Desulfobulbu & 0.00767486 & Lautropia & 0.01163532 & Schwartzia & 0.01497963 & Fusobacteriu & 0.00057786 & Shuttleworth & 0.00617352 \\
\hline Romboutsia & 0.004980457 & 7 Treponema & 0.0258245 & Capnocytoph & h) 0.01863692 & Tannerella & 0.01531352 & Panimonas & 0.00645228 & Peptoniphilus & 0.01131323 \\
\hline Catonella & 0.009130838 & 8 Achromobac & 0.04165186 & ella & 0.01973323 & Treponema & 0.01586972 & Campylobact & 0.0128947 & & 0.01399269 \\
\hline Saccharibact & 0.013648 & Ild & [0.0301. & or & 0.03337993 & Neisseria & 0.01607126 & Romboutsia & 0.01376227 & Cryptobacter & 10.024 \\
\hline Fusobacteriu & 0.017850754 & vartzia & 0.06380201 & Enterococcus & 0.03445 & oniphllus & 0.0 & acteriv & 0.01485281 & Granulicatell & 0.0378569 \\
\hline Peptostrepto & 0.032861 & 8 Pyramidobad & 0.0726406 & Escherichia/s & 0,03862957 & Scardovia & 0.03884765 & Rothia & 0,02025476 & Lautropia & 0.04181737 \\
\hline Enterococcus & 0.043417378 & 8 Escherichia/s & 0.08075315 & Deinococcus & 80.04597273 & Raoultella & 0.03991252 & Blautia & 0.02662666 & Roseburia & 0.04285195 \\
\hline Campylobact & 0.043509591 & 1 Propionibact & 0.08388968 & Slackia & 0.05350477 & Comamonas & 0.04260764 & Cryptobacter & If 0.02893532 & Niveispirillum & 0.04341788 \\
\hline Iobacteriu| & 0.048392 & $m_{1}$ & 0.084440 & 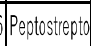 & 0.05612149 & ctor & 0.04531431 & Mogiba & 0931400 & Catonella & 0.04462881 \\
\hline
\end{tabular}

\begin{tabular}{|c|c|c|c|c|c|}
\hline \multicolumn{5}{|c|}{ Gut Microbiome (genus, $p$-value) } \\
\hline \multicolumn{2}{|c|}{ Male and Female } & \multicolumn{2}{c|}{ Male } & \multicolumn{2}{c|}{ Female } \\
\hline AMY1A & AMY2A & AMY1A & AMY2A & AMY1A & AMY2A \\
\hline
\end{tabular}

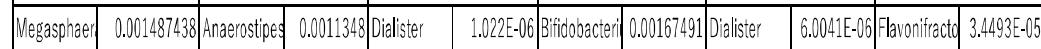

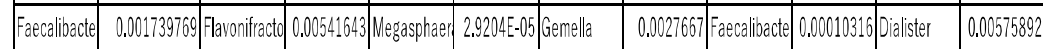

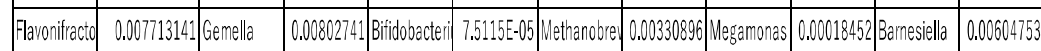

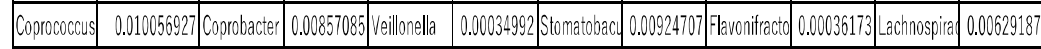

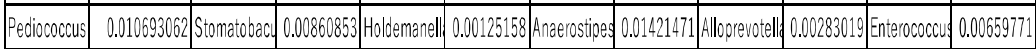

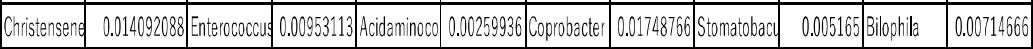

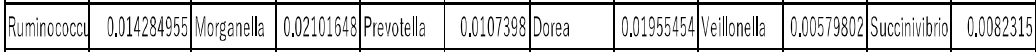

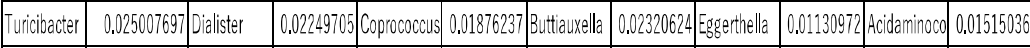

\begin{tabular}{|l|l|l|l|l|l|l|l|l|}
\hline Egserthella & 0.039521963 & Bilophila & 0.02732775 & Olsenella & 0.02155837 & Megamonas & 0.02544681 & Clostricium X 0.01850922 Anaerofustis 0.02157995 \\
\hline
\end{tabular}

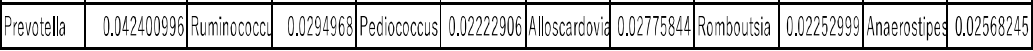

\section{1}

